\title{
Does spiral galaxy IC 342 exhibit shear induced wave transformations!?
}

\author{
S. Poedts ${ }^{1, \star}$ and A. D. Rogava ${ }^{2,3,4}$ \\ 1 Centre for Plasma Astrophysics, K.U.Leuven, Celestijnenlaan 200B, 3001 Leuven, Belgium \\ 2 Abastumani Astrophysical Observatory, Kazbegi ave. $2^{a}$, Tbilisi-380060, Georgia \\ 3 Tbilisi State University, Chavchavadze ave. 2, Tbilisi-380028, Georgia \\ 4 Abdus Salam International Centre for Theoretical Physics, 34014 Trieste, Italy
}

Received 22 June 2000 / Accepted 15 January 2002

Abstract. In this paper we argue that the peculiar magnetic spiral structure of the giant, face-on spiral galaxy
IC 342 may be evidence for velocity shear induced magnetohydrodynamic (MHD) density wave transformations.

Key words. magnetohydrodynamics (MHD) - waves - galaxies: individual: IC 342 - galaxies: kinematics and dynamics - galaxies: magnetic fields

\section{Introduction}

The universal appearance of shear flows in astrophysics implies that the velocity shear induced "nonmodal" processes (Trefethen et al. 1993), associated with the non-selfadjoint character of the governing linear dynamics, may substantially influence the physics of the corresponding astrophysical objects which may explain their different and remarkable observational appearance. One notable nonmodal process, that was recently disclosed, (Chagelishvili et al. 1996), is associated with shear flows sustaining more than one mode of wave motion. In such systems the shear-induced drift of the wave vector $\boldsymbol{k}(t)$ evokes a temporal variation of the eigenfrequencies (Chagelishvili et al. 1994) and leads to a shear-induced coupling of the corresponding wave modes maintained by the "ambient" shear flow. Under favorable physical conditions the shearinduced coupling leads to mutual transformations of different waves into each other.

The advantage of the nonmodal approach is that in many interesting astrophysical situations it makes it possible to reduce the complicated mathematical layout of the problem to the solution of a relatively simple and solvable set of ordinary differential equations (ODEs). In the case of wave transformation processes it is also possible to further reduce this set of ODEs to a pair of second order, ordinary differential equations describing the dynamics of two coupled linear oscillators. The term "cou-

Send offprint requests to: S. Poedts,

e-mail: StefaanPoedts@wis.kuleuven.ac.be

* Research Associate of the Belgian National Fund for Scientific Research (FWO-Vlaanderen). pled oscillations" refers to the case where two (or more) oscillators, on equal footing, are coupled (e.g. by a set of springs) so that the motion of each of them is affected by the other(s). This remarkable common mathematical basis with the well-known oscillatory system of Classical Mechanics helps to understand qualitatively the wave interaction processes in fluid mechanics and plasma physics and in all those astrophysical instances, where these processes presumably appear (Rogava et al. 2000).

This kind of mechanism (hereafter referred as shearinduced transformations or SITs) may be operational in a wide variety of astrophysical objects. In a pulsar magnetosphere, for example, it may ensure the conversion of nonescaping Langmuir oscillations, maintained by the highly relativistic magnetospheric electron-positron plasma, into the escaping radio emission of pulsars (Mahajan et al. 1997). In the solar atmosphere it may account for the generation of solar wind MHD waves, for the heating of the solar corona and the acceleration of the solar wind (Poedts et al. 1998, 1999; Rogava et al. 2000). Other potential astrophysical applications (see the discussion of Mahajan \& Rogava 1999) include planetary atmospheres and interiors, planetary rings, stellar atmospheres, various kinds of accretion flows (accretion disks, disk winds, accretion columns), jets in AGN's, etc. In other words, in all astrophysical objects where some kind of shear flow is present and where the flow can sustain two or more modes of wave motion, velocity shear-induced wave transformations are very likely to occur.

Another prominent astrophysical example of a magnetized astrophysical shear flow is a galactic gaseous disk. Fan and Lou recently demonstrated that galactic 
discs can nourish both slow $\left(\sim 0.2 \mathrm{~km} \mathrm{~s}^{-1}\right)$ and fast $\left(\sim 20 \mathrm{~km} \mathrm{~s}^{-1}\right)$ MHD density waves (Fan \& Lou 1996, 1997). Moreover, their numerical results contained strong evidence for the existence of coupling between these two modes of MHD density waves. Recently it was explicitly confirmed (Rogava et al. 1999) that the coupling actually exists, that it is exclusively due to the presence of the velocity shear and that it can lead to mutual transformations of the waves into each other.

It is a fascinating challenge to disclose the first observational evidence of velocity-shear-induced transformations over several Megaparsecs, in some of the nearby spiral galaxies! The aim of this paper is to argue that the giant, face-on spiral galaxy IC 342 (from the Maffei1 subgroup of the Local Group of galaxies) may be the first astrophysical object where one can find the experimental evidence for the velocity shear induced wave transformations.

\section{Main consideration}

The physical theory of the large-scale structure of spiral galaxies is based on the well-known density wave scenario. The stellar disk is mainly visible in red and near infrared bands, contains relatively old stars and sustains moderate strength, large-scale density wave structures. Thermal and magnetic energy densities are of comparable magnitude: for typical nearby galaxies (such as M51, NGC 6946 and IC 342$)$, the Alfvén $\left(C_{\mathrm{A}}\right)$ and sound $\left(C_{\mathrm{s}}\right)$ speeds are comparable and fall in the range $\sim 10-20 \mathrm{~km} \mathrm{~s}^{-1}$. This pledges the existence of both fast $\left(\sim 20 \mathrm{~km} \mathrm{~s}^{-1}\right)$ and slow $\left(\sim 0.2 \mathrm{~km} \mathrm{~s}^{-1}\right)$ MHD density waves in such a disk system. An important feature of MHD density waves, which helps to distinguish them from one another, concerns the phase characteristics of mass density and azimuthal magnetic field perturbations (Fan \& Lou 1996, 1997). In particular, for fast MHD density waves the enhancement of the surface gas mass density and the parallel component of the magnetic field are more or less in phase, while for slow MHD density waves there is a significant $(\geq \pi / 2)$ phase difference between the mass density and the azimuthal magnetic field perturbations.

Fan and Lou applied this criterion in the analysis of large-scale structures within nearby individual spiral galaxies. The "Whirlpool galaxy" M51, for example, has been identified as a clear case of a galactic gaseous disk with fast MHD density waves. Recently, these authors performed an analogous study for the spiral galaxy NGC 2997 which revealed its close resemblance with the M51 case (Fan \& Lou 1999). Moreover, since according to the MHDdensity-wave scenario the spiral pattern of NGC 2997 is identified with fast MHD density waves, Fan and Lou were able to predict the existence of a (still to be discovered) moderate H1 gas arm associated with the "magnetic arm" in the radio continuum.

Recently, it was discovered that there is another possible class of late-type, gas-rich spiral galaxies with coherent large-scale magnetic spiral arms located between the optical spiral arms (NGC 6946 is an example of this class). Both its optical and magnetic spiral arms occupy a disk angular radius of $\sim 4$ arcmin, where the galactic gaseous disk rotates almost rigidly (i.e., the rotation speed $V_{\theta}$ increases almost linearly). Further out the rotation curve slowly bends and approaches a constant value $V_{\theta} \simeq 200 \mathrm{~km} \mathrm{~s}^{-1}$, which remains roughly constant in the radial range from 4-11 arcmin. This fact allowed Fan and Lou to conclude (Fan \& Lou 1999) that the gaseous disk in NGC 6946 maintains slow MHD density waves.

However, there is at least one example of a spiral galaxy where optical and radio continuum observations indicate the presence of both kinds of MHD density waves! This is the spiral galaxy IC 342, seen behind the Milky Way in the Camelopardalis constellation and physically belonging to the Maffei1 subgroup of the Local Group of galaxies. IC 342 is one of the largest galaxies in the northern sky: probably the third largest in apparent size, preceded only by the Andromeda and Triangulum galaxies. It is of the Hubble type Scd with a well developed spiral structure and is almost face on. Its distance is unclear (given by de Vaucouleurs (1979) to be $3.1 \mathrm{Mpc}$ and by Newton $(1980 \mathrm{a}, \mathrm{b})$ to be $4.5 \mathrm{Mpc})$. The rotation velocity $V_{\theta}$ increases linearly with radius up to about $5 \mathrm{kpc}$, beyond which it becomes flat and remains flat as far out as can be established. A remarkable feature of this galaxy is that it has relatively weak density waves between $11 \mathrm{kpc}$ and $15 \mathrm{kpc}$. So it seems that density waves might not have much influence on gas motions within the outer ring of its gaseous disk and the gas can be assumed to be axisymmetrically distributed. The optical spiral structure of IC 342 is predominantly confined to the inner, rigidly rotating region $\left(\leq 10^{\prime}\right)$. The size of the radio-continuum emission region is considerably larger than the optical one (see e.g., Fig. 9 in Grave \& Beck 1988). The results of Krause et al. (1989) imply that ridges of polarized emission in the inner disk are located in the interarm regions of the optical spiral arms. In the outer disk, on the contrary, magnetic arms extend far outside the optical spiral pattern, but $H 1$ is distributed in a symmetric way implying the existence of a physical link between these patterns. Moreover, $H 1$ images show a much larger $\left(\leq 35^{\prime}\right)$ spiral pattern that extends into the disk portion with a largely flat rotation curve. On the basis of the above observational evidence Fan \& Lou (1999) suggested that IC 342 seems to feature both kinds of MHD density waves! In particular, it exhibits slow MHD density waves in the inner (rigidly rotating) region of the galactic gaseous disk and fast MHD density waves in the outer (differentially rotating) region. However, it must be noted that the positioning of polarization ridges may be related to processes of compression and shear induced by optical arm gas flows and/or with the lack of homogeneity of turbulent diffusion in the disk (Rohde \& Elstner 1998; Elstner et al. 2000).

Recently, inspired by the Fan \& Lou theory of galactic MHD density waves (Fan \& Lou 1997, hereafter referred as FL97), we reconsidered their mathematical model for the study of local perturbations in galactic gaseous disks (Rogava et al. 1999). 
In FL97 the authors consider a thin, differentially rotating gaseous disc, embedded in a large-scale azimuthal magnetic field and sustained by its self-gravity. The equilibrium state of the system was specified by the constant surface mass density $\sigma_{0}$, the constant speed of sound $C_{\mathrm{s}}$, the constant vectors of the angular rotation velocity $\boldsymbol{\Omega} \equiv$ $(0,0, \Omega<0)$, the large-scale background azimuthal field $\boldsymbol{B}_{0} \equiv\left(0, B_{0}, 0\right)$, and by the linearized (locally planeparallel) mean velocity field $\boldsymbol{V}_{0} \equiv(0,2 \mathcal{A} x, 0)$, with $\mathcal{A} \equiv(r / 2) \partial_{r} \Omega>0$ and $\mathcal{B} \equiv \Omega+\mathcal{A}$ the first and the second Oort constants, respectively. In the present paper we adopt and use the same equilibrium model.

The excitation and time evolution of compressible MHD perturbations in this system are governed by the set of equations $\left[\mathcal{D}_{t} \equiv \partial_{t}+2 \mathcal{A} x \partial_{y}\right]$ :

$$
\mathcal{D}_{t} \sigma+\sigma_{0}\left(\partial_{x} u+\partial_{y} v\right)=0
$$

$\mathcal{D}_{t} u-2 \Omega v=-\partial_{x} \chi+\frac{B_{0}}{4 \pi \rho_{0}}\left(\partial_{y} b_{x}-\partial_{x} b_{y}\right)$,

$\mathcal{D}_{t} v+2 \mathcal{B} u=-\partial_{y} \chi$,

$\mathcal{D}_{t} b_{x}=B_{0} \partial_{y} u$,

$\partial_{x} b_{x}+\partial_{y} b_{y}=0$

where $\sigma, u, b_{x}, v, b_{y}, \psi$ are the perturbations of surface mass density, velocity and magnetic field radial and azimuthal components and gravitational potential, respectively and $\chi \equiv C_{s}^{2} \sigma / \sigma_{0}+\psi$. These equations constitute a closed set together with the three-dimensional Poisson's equation: $\left(\partial_{x}^{2}+\partial_{y}^{2}+\partial_{z}^{2}\right) \psi=4 \pi G \sigma \delta(z)$, where $G$ is the gravitational constant and $\delta(z)$ is the Dirac delta function in terms of the vertical coordinate $z$.

In this case, Kelvin's transformation of variables $x^{\prime}=$ $x, y^{\prime}=y-2 \mathcal{A} x t, z^{\prime}=z, t^{\prime}=t$ is sufficient to recover the class of nonexponentially evolving perturbations that is overlooked in the framework of the standard normal-mode approach. The switch to these variables transforms the initial spatial inhomogeneity of the system (1-5) to a time inhomogeneity, because $\mathcal{D}_{t} \rightarrow \partial_{t^{\prime}}$ and $\partial_{x} \rightarrow \partial_{x^{\prime}}-2 \mathcal{A} t^{\prime} \partial_{y^{\prime}}$. Hence, one can look for solutions in the form of Spatial Fourier Harmonics (SFH) (Chagelishvili et al. 1994), bearing the form $\sim \exp \left[i\left(k_{x^{\prime}} x^{\prime}+k_{y^{\prime}} y^{\prime}\right)\right]$, where $k_{x^{\prime}}$ and $k_{y^{\prime}}$ are components of the wavenumber vector. Employing this ansatz one can effectively reduce the system to a set of first order ordinary differential equations with time-dependent coefficients (Fan \& Lou 1997) for the perturbation amplitudes $\left(\hat{\sigma}, \hat{u}, \hat{b_{x}}, \hat{v}, \hat{b_{y}}, \hat{\chi}\right)$ of the relevant Fourier components (the superscript ' is hereafter dropped). Moreover, Rogava et al. (1999) proved that this system is further reducible to the following pair of second order ordinary differential equations:

$$
\begin{aligned}
\partial_{\tau}^{2} \mathcal{L}+W_{1}^{2} \mathcal{L} & =2 \omega \partial_{\tau} b+C b, \\
\partial_{\tau}^{2} b+W_{2}^{2} b & =-2 \omega \partial_{\tau} \mathcal{L}+C \mathcal{L},
\end{aligned}
$$

where $b \equiv i b_{x} / B_{0}, \mathcal{S} \equiv i \hat{\sigma} / \sigma_{0}, \mathcal{L} \equiv \mathcal{S}+\beta(\tau) b, W_{1}^{2} \equiv 1+\alpha$, $W_{2}^{2} \equiv \xi^{2}\left(1+\beta^{2}\right)+(1+\alpha) \beta^{2}+4 \omega R, C \equiv(1+\alpha) \beta$. The function $\beta(\tau) \equiv \beta_{0}-2 R \tau$ describes the shear-induced linear drift of the perturbation wave number vector, with $\beta_{0} \equiv k_{x} / k_{y}$ the ratio of the initial values of the wave number components, $\tau \equiv t C_{\mathrm{s}} k_{y}$ the dimensionless time variable, and $R$ the normalized first Oort constant. The parameter $\delta \equiv-2 \pi G \sigma_{0} / C_{\mathrm{s}}^{2} k_{y}$ ( $\sigma_{0}$ being the equilibrium surface mass density) and the function $\alpha(\tau) \equiv \delta /(1+$ $\left.\beta^{2}\right)^{1 / 2}$ result from the presence of self-gravity in the galactic disc. The parameter $\omega \equiv \Omega / C_{\mathrm{s}} k_{y}$ is related to the presence of the rigid rotation component in the total kinematic portrait of the galactic disc. Finally, $\xi \equiv C_{\mathrm{A}} / C_{\mathrm{s}}$ is the ratio of the Alfven speed to the speed of sound.

The physical coupling in the system (5) is exclusively due to the differential character of the motion. This is obvious since only the presence of the shear $(R \neq 0$, leading to $\alpha=\alpha(\tau)$ and $\beta=\beta(\tau))$ makes the coefficients of the system (5) time-dependent (Rogava et al. 1999).

However, the presence of the sheared motion (differential rotation in the galactic gaseous disk) is a necessary but not a sufficient condition for the shear-induced coupling to "inflame" efficient mutual transformations of MHD density waves into each other. For transformations to be efficient it is also necessary that the Alfvén speed $C_{\mathrm{A}}$ and the sound speed $C_{\mathrm{s}}$ have approximately the same magnitude, i.e. $C_{\mathrm{A}} \simeq C_{\mathrm{s}}$. In parallel shear flows, where both self-gravity and rotational effects are absent $(\delta=\omega=0)$, the condition $C_{\mathrm{A}}=C_{\mathrm{s}}$ is a necessary condition for wave transformations to be efficient (Chagelishvili et al. 1996). It is not yet clear whether this criterion remains the same or whether it is somehow affected by the presence of the self-gravitation $(\delta \neq 0)$ and Coriolis parameter $(\omega \neq 0)$ in galactic gaseous discs. But it is known that these physical factors (self-gravity and nonzero Coriolis parameter) lead to the appearance of the transient Jeans instability and "epicyclic shaking", respectively (Fan \& Lou 1997).

A systematic study of this issue is currently in preparation (Poedts \& Rogava 2001) and preliminary results show that condition $C_{\mathrm{A}} \simeq C_{\mathrm{s}}(\xi \simeq 1)$ remains qualitatively valid for the galactic case as well (see below). Different combinations of the system parameters must lead to rather different interferences of the wave transformation and the transient amplification processes, so that the outcome of the whole set of the shear-induced processes in galactic gaseous disks can be diverse. It is obvious that this kind of detailed study for the plausible ranges of involved parameter values is important for developing the quantitative theory.

\section{Results}

To the best of our knowledge, it is impossible to find in the literature any definite information about the values of all those physical parameters for the galaxy IC 342 which are important in the perspective of the efficiency of shear-induced wave transformations. That is why we can hardly make a choice of parameters, closely related to 


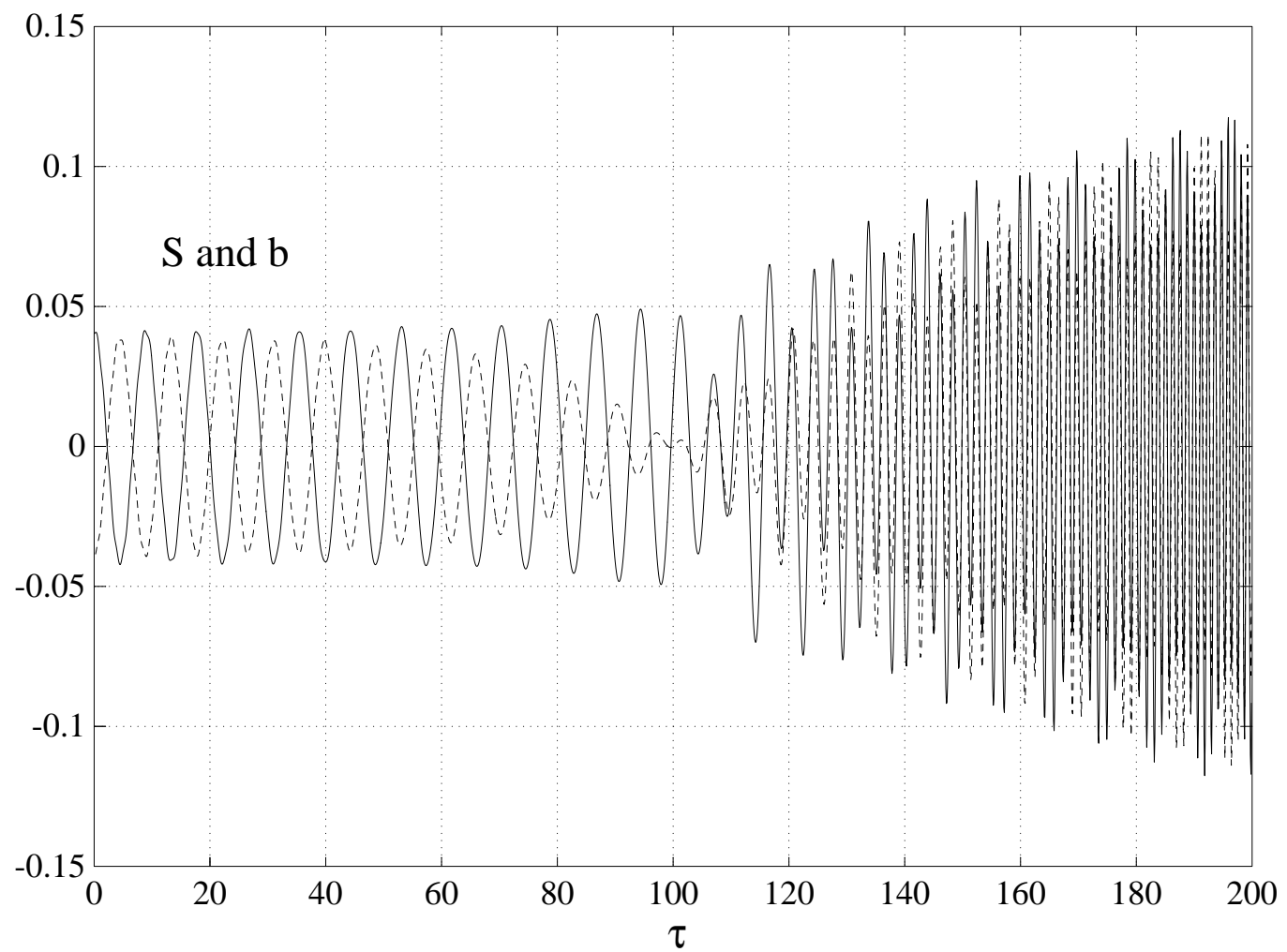

Fig. 1. Temporal evolution of density $(S(\tau)$, solid line) and azimuthal magnetic field $(b(\tau)$, dashed line) perturbations. The plots are drawn for the case when $\beta_{0}=4, R=0.02, \omega=-0.05, \xi=1$, and $\delta=-0.1$.

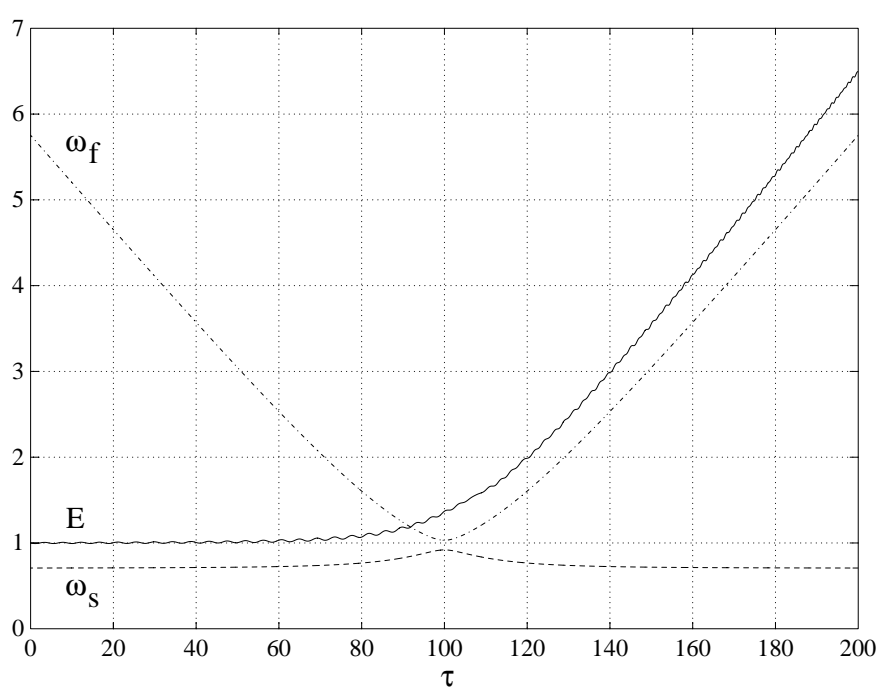

Fig. 2. Evolution of the total energy of the above perturbation $(E(\tau)$, solid line) plotted in the same frame with graphs for the time-dependent dispersion curves of SMW $\left(\omega_{\mathrm{s}}(\tau)\right.$, dashed line) and FMW $\left(\omega_{\mathrm{f}}(\tau)\right.$, dashed-dotted line). The plots are drawn for the case when $\beta_{0}=4, R=0.02, \omega=-0.05, \xi=1$, and $\delta=-0.1$.

the situation in the different regions of this galaxy. Hence, it is still too early to try to develop a concrete model for these processes and to point out possible diagnostics.

Additional uncertainty comes from our current inability to guess the identity and location of "primary" MHD modes for the further onset of the SITs. Observations suggest, however, that IC342 involves a slow wave in the inner (nearly rigid rotation) region and a fast wave in the outer, differentially rotating region. So if the working hypotheses is that the shear-induced wave transformations are responsible for the presence of both these types of waves, it is reasonable to state that the transformations take place in the outer part, where the flow of the galactic gas is sheared.

Instead of giving a detailed analysis, here we wish to present only the illustrative example of SMW-FMW transformations, which is shown in Figs. 1 and 2. Figure 1 displays the evolution of the density (solid line) and the azimuthal magnetic field (dashed line) perturbations. It is clear that the perturbation is initially a pure SMW with substantial phase difference between these two oscillations and that it is transformed into an almost pure FMW, with a higher frequency and with oscillations of $\mathcal{S}(\tau)$ and $b(\tau)$ which are, now, approximately in phase. The evolution of the total energy of the same perturbation (solid line) is shown in Fig. 2. The $E(\tau)$ graph is plotted together with the time-dependent dispersion curves of the SMW $\left(\omega_{\mathrm{S}}(\tau)\right.$, dashed line) and the FMW $\left(\omega_{\mathrm{f}}(\tau)\right.$, dashed-dotted line) effective normal frequencies. The energy evolution is adiabatic (Chagelishvili et al. 1994; Chagelishvili et al. 1996), following the SMW/FMW dispersion curve before/after the transformation.

For these illustrative plots we deliberately have chosen values of the Coriolis and the self-gravity parameters $(\omega$ and $\delta)$ so that the influence of the "epicyclic shaking" and the transient Jeans instability, respectively, 

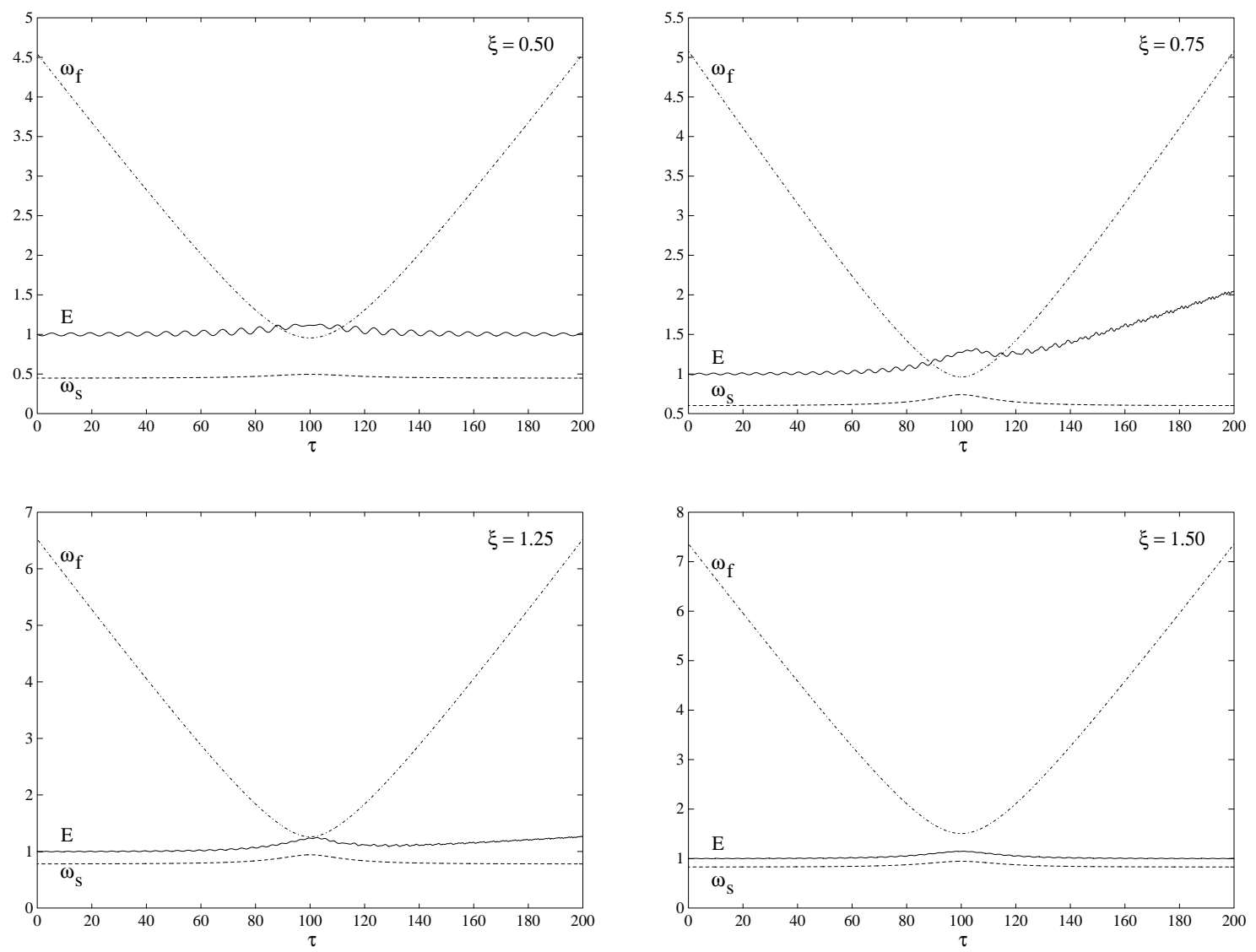

Fig. 3. Evolution of the total energy $(E(\tau)$, solid line) plotted in the same frame with graphs for the time-dependent dispersion curves of SMW $\left(\omega_{\mathrm{s}}(\tau)\right.$, dashed line) and FMW $\left(\omega_{\mathrm{f}}(\tau)\right.$, dashed-dotted line) $\beta_{0}=4, R=0.02, \omega=-0.05$, and $\delta=-0.1$. The values of $\xi$ are equal to: $\xi=0.5$ (upper left), $\xi=0.75$ (upper right), $\xi=1.25$ (lower left), $\xi=1.5$ (lower right), respectively.

would be negligible in comparison with the shear induced transformations. The emergence of these phenomena and their interference with the SITs needs a detailed separate study and is intended to be published elsewhere (Poedts \& Rogava 2001).

The high efficiency of the SITs, visible in Figs. 1 and 2, is guaranteed by the condition $C_{\mathrm{A}}=C_{\mathrm{s}}$ (i.e. $\xi=1$ ), which, as it has been discovered by Chagelishvili et al. (1996), implies the most favorable regime for efficient transformations of SMWs into FMWs. When the ratio of Alfvén and sound speeds is either larger or smaller than one, the SITs become visibly less pronounced. In other words, the wave mode coupling becomes less efficient for $\xi \neq 1$. In order to illustrate the importance of this condition, we made numerical runs for different values of this parameter $\xi=C_{\mathrm{A}} / C_{\mathrm{s}}$, keeping all other parameters fixed at the same values as in Figs. 1 and 2. In Fig. 3 the energy plots (analogous to Fig. 2) are shown for $\xi=0.5,0.75,1.25$, and 1.5 , respectively. One can see from the evolution of the total energy that, when the Alfvén speed is either larger or smaller than the speed of sound, the efficiency of the transformation drastically diminishes. The further we are from the optimum condition $\xi=1$, the more the adiabatic energy graph tends to follow the dispersion curve for the initial SMW mode, i.e., a smaller fraction of the initial SMW is transformed into the FMW.

\section{Discussion and conclusions}

It is a remarkable fact that according to extensive observational data for typical nearby spiral galaxies (including IC 342) the Alfvén speed $C_{\mathrm{A}}$ and the sound speed $C_{\mathrm{s}}$ are indeed of comparable magnitude and fall in the range $\sim 10-20 \mathrm{~km} \mathrm{~s}^{-1}$. This allows us to argue that shear-induced MHD density wave couplings in the magnetized, self-gravitating, differentially rotating gaseous disc of IC 342 should lead to mutual transformations of these waves. The physical picture, as it seems plausible to us, may be the following: slow MHD density waves are excited within the inner (rigidly rotating) region and keep their identity throughout the whole inner disk, since velocity shear is absent and there is no way to transform them into the fast MHD density waves. However, as soon as rotation becomes differential and the flow of the galactic gas becomes sheared, conditions for the shear-induced transformation of "primary" slow MHD density waves into the "secondary" fast MHD waves become favorable, providing the condition $C_{\mathrm{A}} \simeq C_{\mathrm{s}}$ is fulfilled. The efficiency of the transformation is rather sensitive to the value of the ratio $\xi \equiv C_{\mathrm{A}} / C_{\mathrm{s}}$, it is the strongest (almost complete) when $\xi=1$ and becomes less pronounced for either $\xi>1$ and $\xi<1$. The visibility of the transformation phenomenon also depends on whether the physical mechanism 
launching MHD density waves in the rigidly rotating inner disk is producing pure slow MHD density waves or, rather, some mixture of both slow and fast waves. Generally, it is possible that fast and slow MHD density waves coexist in the almost rigidly rotating part of a spiral galaxy. In this case, transformations of both wave modes will still occur in the differentially rotating region of the galactic gaseous disk, but the resulting wave bundle will remain to be a mixture of both magnetoacoustic waves so that the presence of transformations will be hardly visible.

One can also speculate about the possible existence of galaxies where either slow and/or fast MHD density waves might be excited by a central bar (like NGC 6946 which appears to have a weak central bar) or by a satellite galaxy (e.g., M51 and its companion NGC 5195). This means that in different galaxies MHD wave portraits of both rigidly rotating and differentially rotating regions of the galaxy disk and associated MHD wave transformation processes can be widely diverse. It is even possible that contrary to the well-known WKB or tight-winding approximation theories, which do not encompass the effect of mutual wave transformations, there are spiral galaxies with fast MHD density waves in the inner, rigidly rotating disk and slow MHD density waves in the outer, differentially rotating disk. Everything depends on the location and morphology of the sources of "primary" MHD waves, on the specific rotation curves of galaxies and on the values of $\xi$ in differentially rotating regions of galactic disks, where transformations may or may not occur depending on the presence or absence of the $\xi \simeq 1$ condition.

Galactic interstellar medium is resistive, with turbulent magnetic diffusion being about $3 \times 10^{26} \mathrm{~cm}^{2} \mathrm{~s}^{-1}$ (Ruzmaikin et al. 1988; Elstner et al. 2000). Therefore, magnetic diffusion must play an important role in the dynamics of galactic gaseous disks. Certainly the coefficient is not homogeneous throughout the whole disk but varies being higher in spiral arms due to star formation activity and lower in interarm regions. Irrespective of their identity both modes of MHD density waves are subject to damping via magnetic diffusion. But since the optical and radio continuum observations seem to allow the presence of MHD waves in galactic disks, it appears that despite the damping, there are other, probably more robust physical processes, which guarantee the persistence of the wave appearance. In our opinion the SITs can be one of these processes. Unfortunately, currently, we are only at the very beginning of the understanding of the real-space appearance of the SITs. But from numerical simulations for simple, plane-parallel MHD flows (Bodo et al. 2001) we recently learned that the SIT persist even in the presence of sufficiently large magnetic diffusion.

Certainly, a crucial test for any theoretical result or prediction is a comparison with observational data. Since the observational picture of magnetic field in galaxies is so complicated one can not even claim that MHD waves are the unique or even the most important process in spiral galaxies. Most probably the situation is very diverse in different galaxies. In the galaxy M51, for instance, which seems to exhibit the appearance of fast MHD density waves (Fan \& Lou 1999), the structure of the magnetic field can also be well explained by turbulent or fast dynamo mechanisms (Elstner et al. 1990; Hanasz \& Lesch 1998) and/or compressions and shears provoked by the spiral arms (Rohde \& Elstner 1998; Elstner et al. 2000). This means that we, suggesting the SITs as a possible mechanism for the generation of the particular magnetic structure in IC 342, are fully aware that serious proofs in favor of this suggestion can be found only after detailed observational studies. That is why we consider this idea as a cautious hypotheses rather than a direct prediction.

Another efficient way to test the validity of our theoretical model would be the knowledge about the pitch angle picture for the IC 342. The observations of nearby galaxies (Beck \& Hoernes 1996; Ehle et al. 1996) disclose that the magnetic structure is mainly spiral with quite large pitch angles, similar to pitch angles of optical arms. The IC 342 observations give the pitch angle value about $18^{\circ}$ (Krause et al. 1989). Unfortunately, our current model is limited to the study of the temporal evolution of individual wave harmonics, and does not allow to reproduce a picture of the azimuthal distribution for the MHD waves in order to check whether the pitch angles can match the observed value. The latter will become possible only after we will have an adequate numerical model with an accurate realspace portrait of the phenomenon.

It is well-known that about half of the total galactic magnetic field comes in the form of its random component (Ruzmaikin et al. 1988). This poses an interesting question: how does the presence of the random component influence the SITs taking place in galactic gaseous disks?! This question, being a part of the more general problem of the influence of random fields on the shearinduced physical processes, obviously requires a separate and comprehensive consideration. On the basic, theoretical level one needs to learn how to adjust the existing schemes of the nonmodal approach to the study of flows influenced by stochastic fields. Direct numerical investigations are also an option, since after having a numerical model for a gaseous disk with regular equilibrium magnetic field it would be easier to include random components into the model and to test their influence on shearinduced processes in a numerical way.

Therefore, it seems obvious that the hypothesis, argued in this paper, needs to be addressed further. The detailed observations of IC 342, as well as of other, similar kinds of spiral galaxies may give additional evidence in favor of (or contrary to) our interpretation. On the theoretical level the further development of this approach, with the possible inclusion of dissipative effects, random fields and with numerical studies of the "real-space" appearance of MHD density waves, will help to "transform" this hypothesis into a model with definite predictive power.

Acknowledgements. Our work was supported, in part, by the INTAS grant No. 97-0504. A.D.R. wishes to thank Abdus 
Salam International Centre for Theoretical Physics for supporting him, in part, through an Associate Membership Award.

\section{References}

Beck, R., \& Hoernes, P. 1996, Nature, 379, 47

Bodo, G., Poedts, S., Rogava, A. D., \& Rossi, P. 2001, A\&A, 374,337

Chagelishvili, G. D., Rogava, A. D., \& Segal, I. N. 1994, Phys. Rev. E, 50, 4283

Chagelishvili, G. D., Rogava, A. D., \& Tsiklauri, D. G. 1996, Phys. Rev. E, 53, 6028

Ehle, M., Beck, R., Haynes, R. F., et al. 1996, A\&A, 306, 73

Elstner, D., Otmianowska-Mazur, K., von Linden, S., \& Urbanik, M. 2000, A\&A, 357, 129

Elstner, D., Meinel, R., \& Rüdiger, G. 1990, Geophys. Astrophys. Fluid Dyn., 50, 85

Fan, Z. H., \& Lou, Y. Q. 1996, Nature, 383, 800

Fan, Z. H., \& Lou, Y. Q. 1997, MNRAS, 291, 91

Fan, Z. H., \& Lou, Y. Q. 1999, MNRAS, 308, L1

Goldreich, P., \& Lynden-Bell, D. 1965, MNRAS, 130, 125

Grave, R., \& Beck, R. 1988, A\&A, 192, 66

Kelvin Lord (W. Thomson) 1887, Phil. Mag., 24, 188

Krause, M., Hummel, E., \& Beck, R. 1989, A\&A, 217, 4
Hanasz, M., \& Lesch, H. 1993, 278, 561

Mahajan, S. M., Machabeli, G. Z., \& Rogava, A. D. 1997, ApJ, 479, L129

Mahajan, S. M., \& Rogava, A. D. 1999, ApJ, 518,

Newton, K. 1980a, MNRAS, 191, 169

Newton, K. 1980b, MNRAS, 191, 615

Poedts, S., Rogava, A. D., \& Mahajan, S. M. 1998, ApJ, 505, 369

Poedts, S., Rogava, A. D., \& Mahajan, S. M. 1999, Space Sci. Rev., 87, 295

Poedts, S., \& Rogava, A. D. 2001 (in preparation)

Rogava, A. D., Mahajan, S. M., \& Berezhiani, V. I. 1996, Phys. Plasmas, 3, 3545

Rogava, A. D., \& Mahajan, S. M. 1997, Phys. Rev. E, 55, 1185

Rogava, A. D., Poedts, S., \& Heirman, S. 1999, MNRAS, 307, L31

Rogava, A. D., Poedts, S., \& Mahajan, S. M. 2000, A\&A, 354, 749

Rohde, \& Elstner, D. 1998, A\&A, 333, 27

Ruzmaikin, A. A., Shukurov, A. M., \& Sokoloff, D. D. 1988, Magnetic Fields in Astrophysics (Dordrecht: Kluwer)

Trefethen, L. N., Trefethen, A. E., Reddy, S. C., \& Driscoll, T. A. 1993, Science, 261, 578

de Vaucouleurs, G. 1979, ApJ, 227, 729 